\title{
Téoros
}

Revue de recherche en tourisme

\section{Quel avenir pour l'hébergement touristique rural ?}

Le cas de gîtes et des chambres d'hôtes du Massif central

(France)

\section{Jean-François Mamdy, Marion Guillot et Nathalie Disez}

Volume 26, numéro 3, automne 2007

URI : https://id.erudit.org/iderudit/1071008ar

DOI : https://doi.org/10.7202/1071008ar

Aller au sommaire du numéro

Éditeur(s)

Université du Québec à Montréal

ISSN

0712-8657 (imprimé)

1923-2705 (numérique)

Découvrir la revue

Citer cet article

Mamdy, J.-F., Guillot, M. \& Disez, N. (2007). Quel avenir pour l'hébergement touristique rural ? Le cas de gîtes et des chambres d'hôtes du Massif central (France). Téoros, 26(3), 56-66. https://doi.org/10.7202/1071008ar d'utilisation que vous pouvez consulter en ligne. 


\section{Quel avenir pour l'hébergement touristique rural ?}

\section{Le cas de gîtes et des chambres d'hôtes du Massif central (France)}

\section{Jean-François Mamdy, Marion Guillot et Nathalie Disez}

Le succès des formes d'hébergement spécifiques du tourisme rural en France, gîtes ruraux et chambres d'hôtes, ne se dément pas auprès des clientèles touristiques françaises ou européennes. Pourtant les analystes s'interrogent sur l'avenir de ce type d'hébergement, d'une part en raison de mouvements de retrait observés çà et là après une progression continue du parc de gîtes ruraux, d'autre part en raison d'une progression très vive du nombre de chambres d'hôtes, qui risquerait (paradoxalement) de saturer la demande. L'hébergement touristique rural, dans ses formes les plus spécifiques, tient une place capitale dans l'offre de tourisme rural, dont on sait l'importance pour le développement rural (Disez, 1999; Mamdy et al., 2001). On mesure ainsi l'enjeu stratégique d'une connaissance précise des dynamiques à l'œuvre au sein du parc d'hébergement touristique, ainsi que d'une approche prospective, pour éclairer les professionnels et les décideurs du tourisme rural et fonder leurs choix et leurs actions.

Le présent article exploite les analyses et les résultats d'une recherche réalisée de 2003 à 2005 pour le compte de la DATAR du Massif central et de l'observatoire SPOT Auvergne, par l'unité de recherche NFAER / Métafort de l'ÉNITA de Clermont-Ferrand'.

Il convient de rappeler que les pouvoirs publics commanditaires (DATAR) cherchent à accompagner le développement rural de façon efficace. Dans le schéma de pensée actuel, la performance du secteur d'activité passe par une offre de produits et services de qualité, des entreprises compétitives, des professionnels formés. Reste à savoir si l'ensemble de ces concepts est représentatif des

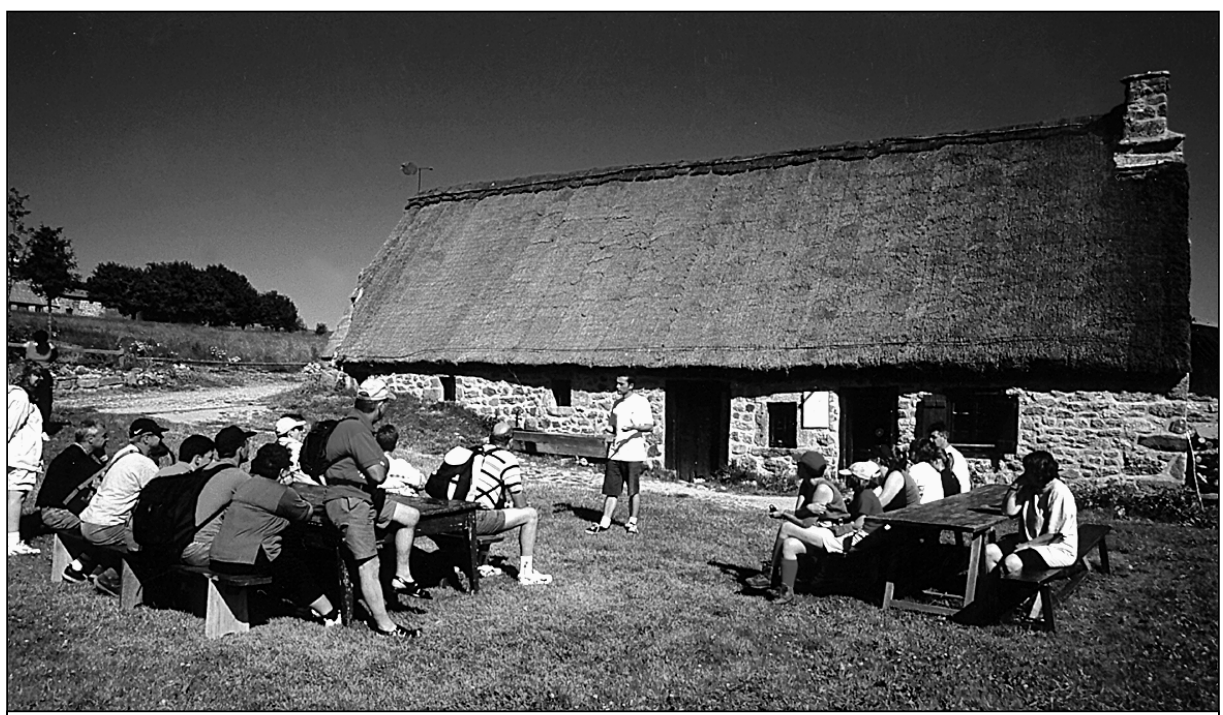

Animation Jasserie du Coq Noir. Parc naturel régional du Livradois Forez.

Source : Route des métiers du Livradois-Forez.

réalités du tourisme rural et pertinent pour l'action... Les gîtes et les chambres d'hôtes sont-ils des produits, au sens où les définissent les spécialistes en marketing, lorsqu'ils parlent du couple produit-marché ? Les structures d'hébergement touristique sont-elles des entreprises au sens d'organisations complexes, assemblant des activités diversifiées, employant souvent du personnel salarié, recherchant le profit? Ou sont-elles des formes d'activité mi-domestique, mi-marchande, majoritairement familiale, consubstantielle d'un projet de vie ? Les hébergeurs, enfin, sont-ils animés de l'esprit d'entreprise ou plutôt d'une passion pour les vieilles pierres? Sont-ils de vrais professionnels du tourisme ou plus généralement des acteurs de la société civile?

Le questionnement sous-jacent à ce travail de recherche-développement, dont la finalité est d'éclairer la décision publique pour accompagner au mieux le développement tou- ristique, s'appuie sur les concepts structurants de l'économie, du marketing et du management (entreprise, produit touristique, professionnalisme), en les soumettant à l'épreuve des faits.

Une première partie introductive décrit le cadre factuel : le parc d'hébergement touristique rural des gîtes et des chambres d'hôtes de la marque "Gîte de France " dans le Massif central et son évolution de 1980 à 2004. Une analyse interprétative des faits est esquissée.

Une deuxième partie affine la connaissance et la compréhension des dynamiques par une investigation auprès des hébergeurs : la distinction de profils d'hébergeurs éclaire des logiques d'acteurs très différenciées.

Se fondant sur l'opinion d'experts, la troisième partie présente un essai prospectif à dix ans sur le parc d'hébergement touristique rural. 
Le propos s'achève par une discussion qui confronte les enseignements des diverses sources d'analyse. Des prévisions et des recommandations sont avancées en fin d'article.

\section{Le parc de gîtes et de chambres d'hôtes : des évolutions différenciées}

Les étapes préliminaires de l'étude ont consisté à retenir un cadre spatial et temporel pour observer l'évolution du parc d'hébergement touristique rural.

Le cadre spatial est celui du Massif central physique, tel que défini par le décret du 20 septembre 1985 l'instituant dans la logique de la Loi Montagne. Le Massif central est un ensemble physique de moyenne montagne habitée, à caractère agricole et rural marqué. L'espace est cependant divers par ses reliefs (montagnes, plateaux, plaines et vallées), ses paysages, ses patrimoines, ses pays ruraux et ses villes. II rassemble de ce fait des espaces, des sites, des territoires à potentiel touristique important, mais plus ou moins mis en valeur et ne constituant pas réellement une destination touristique affirmée.

Le cadre temporel retenu pour l'analyse couvre la période 1980-2004 au cours de laquelle une forte dynamique de développement touristique a marqué l'espace rural. Des dates de référence ont été retenues pour rythmer l'observation des évolutions.

II aurait été souhaitable de prendre en considération toutes les formes d'hébergement spécifiques du tourisme rural. Les difficultés de la collecte d'information ont limité les ambitions aux seuls hébergements labellisés, ignorant donc le parc des meublés locatifs, déclarés ou non. Parmi les hébergements labellisés, seuls les plus spécifiques, mais aussi largement dominants, les Gîtes de France, ont été retenus ${ }^{2}$.

L'information, collectée à l'échelle départementale à partir des brochures, des relais départementaux ou de la fédération nationale, a été traitée sur la base d'indicateurs simples à mobiliser et agrégeables: effectifs de structures, de propriétaires, de chambres, niveaux de qualité.

L'état des lieux général en 2000 (tableau 1), avec 7889 gîtes et 3627 chambres d'hôtes,

Tableau 1

État des lieux du parc d'hébergement en gîtes et tables d'hôtes du Massif central

\begin{tabular}{|c|c|c|c|c|c|c|c|c|}
\hline & $\begin{array}{c}\mathbf{N}^{\text {bre }} \mathbf{G R} \\
\mathbf{1 9 8 0}\end{array}$ & $\begin{array}{c}\mathbf{N}^{\text {bre }} \mathbf{G R} \\
\mathbf{1 9 9 0}\end{array}$ & $\begin{array}{c}\mathbf{N}^{\text {bre }} \mathbf{G R} \\
\mathbf{2 0 0 0}\end{array}$ & $\begin{array}{c}\mathbf{N}^{\text {bre }} \mathbf{G R} \\
\mathbf{2 0 0 4}\end{array}$ & $\begin{array}{c}\mathbf{N}^{\text {bre }} \mathbf{C H} \\
\mathbf{1 9 8 0}\end{array}$ & $\begin{array}{c}\mathbf{N}^{\text {bre }} \mathbf{C H} \\
\mathbf{1 9 9 0}\end{array}$ & $\begin{array}{c}\mathbf{N}^{\text {bre }} \mathbf{C H} \\
\mathbf{2 0 0 0}\end{array}$ & $\begin{array}{c}\mathbf{N}^{\text {bre }} \mathbf{C H} \\
\mathbf{2 0 0 4}\end{array}$ \\
\hline $\begin{array}{l}\text { Massif } \\
\text { central }\end{array}$ & $5119^{\star}$ & 7403 & 7889 & 7615 & $541^{\star *}$ & 1365 & 3627 & 4159 \\
\hline
\end{tabular}

GR - gites ruraux ; $\mathrm{CH}$ - chambres d'hôtes.

* Sans l'Hérault.

** Sans l'Hérault, le Rhône et la Lozère (manque de données).

Source : nos calculs (par agrégation des données des relais départementaux des gîtes de France).

montre l'importance relative du parc d'hébergement touristique du Massif central dans l'ensemble national: respectivement $18 \%$ et $15 \%$ du total. Les hébergements sont répartis dans l'espace avec des variations de densité importantes: le sud du Massif est mieux doté en gîtes, la frange de l'est est mieux pourvue en chambres. Quelques départements sont particulièrement riches en hébergements touristiques ruraux, tels le Lot ou l'Ardèche.

Le principal constat d'évolution pour le parc de gîtes est celui d'une progression d'abord vigoureuse dans la décennie 1980, puis atténuée dans la décennie 1990, et d'un léger recul depuis 2000. À l'inverse, le parc de chambres d'hôtes progresse d'abord lentement dans la décennie 1990, puis très fortement ensuite (tableau 1, graphique 1).

Ces deux trajectoires inversées traduiraientelles un processus de substitution entre un mode d'hébergement ancien désormais démodé et un nouveau concept en progression? L'argumentaire simpliste ne tient pas. Dans sa thèse, Salma Loudiyi (2003) fournit une analyse des faits infiniment plus dé- taillée à l'échelle communale et montre, dans l'imbroglio des dynamiques localisées, la complexité des processus à l'œuvre. Plus simplement, nos analyses montrent la diversité des situations et la particularité des trajectoires départementales et laissent suspecter l'influence des politiques départementales d'appui au développement de l'hébergement touristique. Ainsi, la diminution de l'effectif observé de gîtes ruraux en Ardèche ou dans le Puy-de-Dôme est principalement due à une politique plus sélective des relais. En outre, l'élévation du niveau de qualité des hébergements touristiques creusois est attribuable à la politique incitative mise en place dans le département.

Dans l'ordre des constats globaux d'évolution, il convient de souligner l'élévation générale du niveau de qualité depuis deux décennies, qui porte à près de $40 \%$ le parc de gîtes trois épis et à près de 60 \% le parc de chambres d'hôtes trois épis en 2000. La progression se poursuit du reste puisque, en 2004, 74 \% des chambres d'hôtes Gîtes de France du Massif central sont classées au moins trois épis.

\section{Graphique 1}

Évolution du nombre de gîtes ruraux et de chambres d'hôtes dans le Massif central de 1980 à 2004

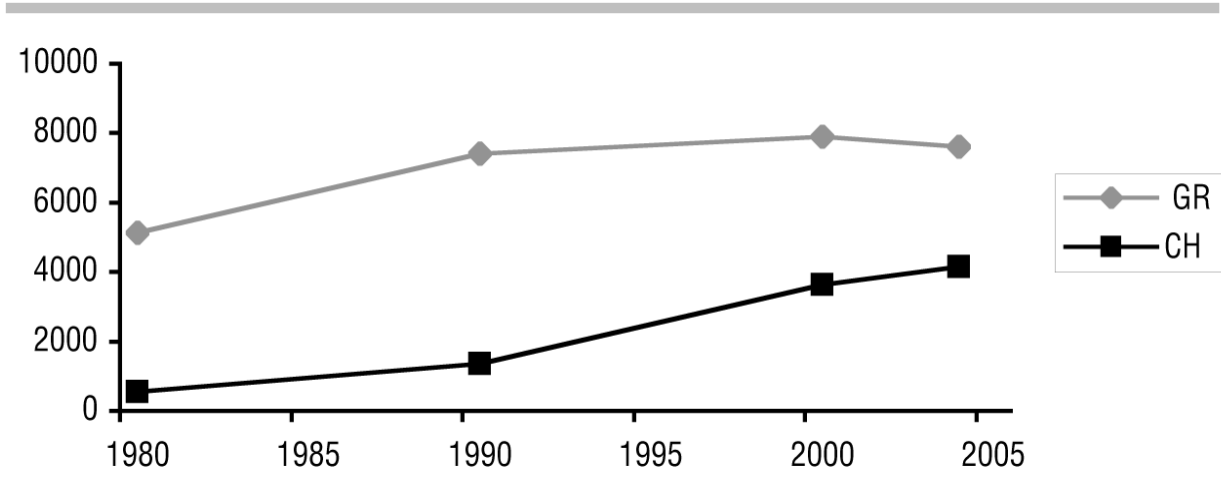

Source : nos calculs (par agrégation des données des relais départementaux des gîtes de France). 
En définitive, le parc d'hébergement touristique rural, globalement réparti sur l'ensemble du Massif, avec des dotations inégales certes selon les départements, semble assez stabilisé pour les gîtes, mais en forte progression pour les chambres. La qualité des hébergements est partout en progression. Aucune loi générale d'évolution spatiale ne se dégage, ni même de différenciation entre dynamique des gîtes et des chambres, si ce n'est l'influence perceptible de l'organisation départementale.

\section{Populations et profils d'hébergeurs touristiques}

Mieux comprendre les dynamiques d'évolution au sein du parc d'hébergement touristique rural suppose de poursuivre l'investigation au-delà des données chiffrées, auprès des propriétaires gestionnaires de structures.

Une hypothèse vraisemblable est que l'on peut distinguer dans la population des acteurs plus ou moins entreprenants, se regroupant en profils de gestionnaires, caractérisés par des pratiques touristiques, avec des logiques et des motivations et, pourquoi pas, des projections spécifiques en termes d'avenir.

Une autre hypothèse est de considérer qu'un distinguo important caractérise les propriétaires gestionnaires de gîtes et de chambres, les premiers répondant à un profil conservateur en lien avec la contraction du parc de gîtes observé, les seconds, plus dynamiques, constituant une population d'acteurs pionniers.

La question des stratégies d'avenir est essentielle dans la perspective de l'étude. Au vu du discours des acteurs interrogés, quelle est la tendance générale et/ou particulière aux différents profils : retrait, continuation ou expansion?

La méthodologie de recherche s'articule en trois étapes :

- Une première phase qualitative privilégie l'entretien approfondi semi-directif auprès d'hébergeurs. L'analyse du discours enregistré permet de relever les pratiques et les justifications sur la base d'une grille inspirée de la théorie des grandeurs (Boltansky et Thévenot, 1991 et 1998) et de construire, en référence à des typologies éprouvées par la recherche
(Disez, 1996 Simmoneaux, 1999 Simon, 2000 Perret et Marcepoil, 2001 ; Charron, 2003), des profils différenciés d'hébergeurs. Un échantillon de 31 structures représentatives de la diversité (localisation, chambres ou/et gîtes, autochtone / nouveau-venu, en activité / en projet / sortant) est enquêté (Gallo, 2004) ; il permet de construire une typologie en cinq classes.

- Une deuxième phase quantitative donne une estimation du poids respectif des profils d'hébergeurs. La méthode utilisée est alors l'enquête téléphonique conduite de façon directive sur la base des profils précédemment construits. L'échantillon comporte 300 propriétaires gestionnaires. II tient compte de la répartition et de la pondération par département, selon la méthode des quotas. Construit par tirage aléatoire (1 pour 20) au sein des registres des relais départementaux, il est représentatif de la population des hébergeurs du Massif central. Le questionnaire reprend la structuration de l'entretien en tête-àtête, mais les questions sont formulées de façon plus directive. Le questionnaire identifie les motivations en référence à celles qui caractérisent les profils de la phase qualitative, les pratiques du prestataire dans son activité touristique, sa posture face à l'avenir. Le codage des réponses permet a priori de trancher sur le profil d'appartenance et de procéder au regroupement.

- Une troisième phase approfondit l'investigation par une analyse spatiale dynamique. En suivant chaque structure d'hébergeur localement, année par année, on cerne plus finement les évolutions que par l'analyse des soldes. Une approche sélective sur les années 2000 à 2004, à l'échelle de plusieurs départements, est testée.

Après un cadrage général de la population d'hébergeurs touristiques, les cinq profils d'acteurs sont analysés. Un essai de quantification est proposé. Une réflexion sur les hypothèses initiales termine l'investigation.

\section{La population d'hébergeurs dans son ensemble}

Les caractéristiques de la population d'hébergeurs touristiques, gestionnaires de gîtes et de chambres d'hôtes, ont été déterminées à partir d'un questionnaire adminis- tré par enquête téléphonique auprès des propriétaires gestionnaires. L'échantillon concerne 300 hébergeurs sélectionnés par la méthode des quotas, par département et type d'activité, à partir des listes des guides Gîtes de France du Massif central (60 \% gîtes, 32 \% chambres, 8 \% gîtes et chambres).

Globalement les hébergeurs sont âgés, la moyenne d'âge étant de 60 ans. La majorité est retraitée. Leur structure d'accueil est de petite dimension : une structure sur deux offre un gîte ou une chambre. Un propriétaire sur cing est agriculteur. Le niveau de formation en tourisme est très faible, puisque seulement $30 \%$ des personnes ont suivi une formation ad hoc. Les deux tiers des prestataires n'ont jamais fréquenté un gîte ou une chambre à titre touristique!

\section{Cinq profils d'hébergeurs différenciés}

Sur les 31 "cas d'étude» analysés, cinq profils d'hébergeurs ont pu être distingués.

\section{Complément de revenu et complément de liens}

Un premier profil rassemble des personnes cherchant dans l'activité touristique des revenus complémentaires: "Comme je prends ma retraite dans six mois et que je n'aurai pas une retraite conséquente, je me suis dit : pourquoi pas... ? Sans l'activité chambres d'hôtes je pense que la retraite me ferait peur. " L'activité d'hébergement au domicile permet aussi de maintenir des liens sociaux dans un environnement de campagne isolée: " J'ai été habituée à voir des gens... donc j'avais peur de cette coupure... de ne voir personne l'hiver. »

Le partenariat professionnel se limite au réseau Gîtes de France et localement à l'Office de tourisme. La communication est gérée a minima.

L'avenir est la poursuite de l'activité dans la continuité : "Au début, on aurait aimé louer davantage... cela s'équilibre. Si on avait fait moins, il aurait fallu faire quelque chose... mais quoi ? " 


\section{Patrimoine : une histoire d'amour}

Il y a dans ce profil une passion des vieilles pierres, un goût pour restaurer le patrimoine et en raconter l'histoire : "Pour la petite chaumière, je passerai d'abord par la fondation du patrimoine. » " J'ai dans l'idée de valoriser des vieilles lettres que j'ai retrouvées, écrites par des gens de là-haut, et qui laissent imaginer comment on vivait il y a 70 ans. »

Ces personnes, principalement concernées par des gites ruraux, sont dans une logique de projet, un projet-passion tourné vers la restauration du patrimoine bâti.

Ici encore la dimension touristique reste complémentaire du projet. Les relations professionnelles sont limitées (Gîtes de France, Office de tourisme).

L'avenir est dans le projet patrimonial et familial : "Je pense que si nous avons des enfants qui nous succèdent ici, ils auront tous les bâtiments qui permettent de faire deux gîtes ruraux dans la propriété. »

\section{Patrimoine: un plan d'épargne dans la pierre}

À la différence du profil précédent, ces hébergeurs visent le placement financier: "Nous avons fait notre partage, nous avons trois maisons et trois enfants... nous préférons leur laisser des maisons que de l'argent. »

Le gîte rural est considéré comme un meublé de location plus qu'un outil de production touristique et l'accueil n'a pas vraiment le statut d'une activité professionnelle: "Vous savez, les Hollandais ont un langage très compliqué, par contre ils sont propres... alors que les Français... »

Ces hébergeurs ne se projettent pas dans un avenir professionnel et pourront assez facilement glisser vers la location à l'année, jugée moins contraignante.

\section{Projet de vie : du temps pour soi}

Cette catégorie choisit l'hébergement touristique à la campagne comme élément d'un projet de vie : " II arrive un moment où l'on aspire à autre chose. Donc, on a cherché une méthode pour changer un peu de style de vie; on avait envie de revenir en

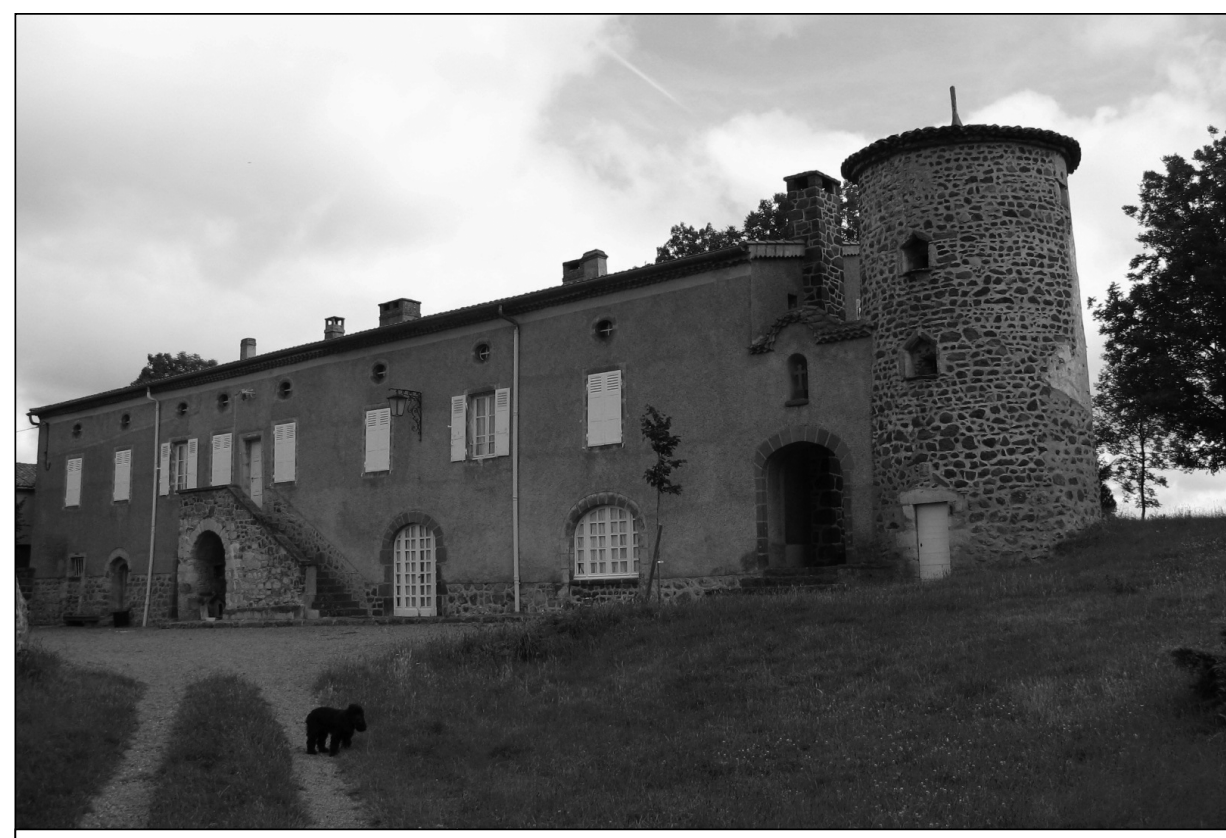

Gîte de groupe. Domaine de Chagourdat dans le Puy de Dôme.

Source : Route des métiers du Livradois-Forez.

Ardèche. Un retour aux sources en fait. » « II y avait une qualité de vie que l'on recherchait, c'était vivre à la campagne, pour avoir la tranquillité, la sérénité. »

Le projet professionnel s'articule au projet de vie, lui étant cependant subordonné : « Moi, j'avais envisagé un moment de faire la table d'hôte, mais bon... étant seule, mon mari travaillant, ça me paraissait un peu lourd. Ce qu'on voulait absolument, c'est garder un petit peu de disponibilité. »

Ces hébergeurs ont un comportement volontariste et professionnel ; ils ne lésinent pas sur l'effort de promotion, utilisant toute une panoplie de moyens variés: cartes de visite, brochures, presse, site Web... Ils sont le plus souvent militants du développement local: " J'ai animé des séances de danses folkloriques..., on a cherché à créer une chorale..., dans notre groupe de développement local on se disait que l'on pouvait faire des choses... Je voulais peut-être faire la démonstration qu'on pouvait faire quelque chose dans le tourisme. »

\section{Projet de vie et d'entreprise}

II s'agit encore ici d'acteurs volontaristes qui ont fait un choix radical en s'installant à la campagne pour créer une entreprise. Leur projet de vie est un projet professionnel : " Je voulais être indépendante, et puis j'avais un bien...
C'est l'opportunité et puis l'envie de revenir à mes sources... Je cherche la rentabilité mais je veux me faire plaisir ! J'ai quitté l'administration pour ça! Je ne supporte pas l'autorité, mais le travail ne me fait pas peur! »

Le professionnalisme rime avec efficacité et rentabilité: "Je veux faire monter ça; de toute façon, je me suis investie comme si je m'investissais dans un commerce. Donc, mes quatre chambres et mon gîte, il faut que ça tourne huit mois dans l'année. »

II s'agit désormais d'une démarche d'entreprise, complexe, évolutive, tirée par la qualité : "Deux gîtes de séjour, quatre chambres d'hôtes trois épis, la ferme-auberge et la ferme pédagogique. On a fait nos quatre chambres dès le départ en 1983, on y a rajouté la table d'hôte en 1995, cela roule bien..., on a travaillé tout de suite sur une nourriture de qualité. Cela s'est su et on a glissé vers la ferme-auberge en 1997. » "J'aurais beaucoup aimé faire, peut-être une fois par mois mais hors-saison, des soirées à thème: des soirées contes, des soirées musique classique, musique de pays... »

Ces personnes sont à la recherche d'innovation et recourent à la formation : " Je prends des cours d'anglais à la chambre de commerce; je me suis perfectionné en l'informatique, je suis prêt à créer mon propre site. » 


\section{Le monde des hébergeurs touristiques change en se diversifiant}

En conclusion, l'analyse du discours a permis de différencier cinq profils contrastés en termes de motivation, de logiques d'action et de pratiques. Le nuancier est intéressant dans la mesure où il renouvelle et enrichit les typologies mises en lumière dans la décennie 1990 : complément de revenu, patrimoine, entreprise (Disez, 1996). La catégorie «projet de vie " dans sa version «temps pour soi » correspond à une nouvelle génération d'acteurs qui organisent leur vie en fin de carrière professionnelle sur un mode midomestique, mi-professionnel. De même, le groupe " patrimoine-épargne » se singularise à la marge de l'activité économique et du placement sécuritaire.

La spécificité de ces deux profils, qui n'avait pas été relevée jusqu'alors à notre connaissance, tient probablement à l'émergence de nouveaux comportements : d'une part, depuis quinze ans, un processus de migration significatif de familles urbaines relativement aisées vers la campagne, attirées par la cadre de vie et décidées à porter un projet de vie en rupture avec leur passé, d'autre part un engouement pour le placement financier dans la pierre, qui s'avère être depuis une dizaine d'années la meilleure garantie contre l'érosion monétaire. Que de tels comportements aient existé autrefois est probable, leur révélation dans le panorama de la diversité des hébergeurs touristiques aujourd'hui en souligne l'importance.

La différenciation en profils souligne I'hétérogénéité des structures d'hébergement touristique et des populations d'hébergeurs. On ne reconnaîtra guère l'archétype de l'entreprise et de l'entrepreneur, sinon dans le dernier profil décrit comme « projet de vie et d'entreprise». Deux autres profils présentent une dimension économique affirmée (complément de revenu et placement financier); pour autant l'activité touristique reste complémentaire et/ou sécuritaire et ne constitue pas une "pure » entreprise... Enfin, les deux derniers profils (patrimoine - passion et projet de vie - temps pour soi) illustrent des modèles d'organisation domestique qui répondent principalement à des finalités sociales et culturelles.

Gîtes ou chambres d'hôtes constituent, nous le pensons, des produits bien différents. Cependant, leur rattachement à tel ou tel

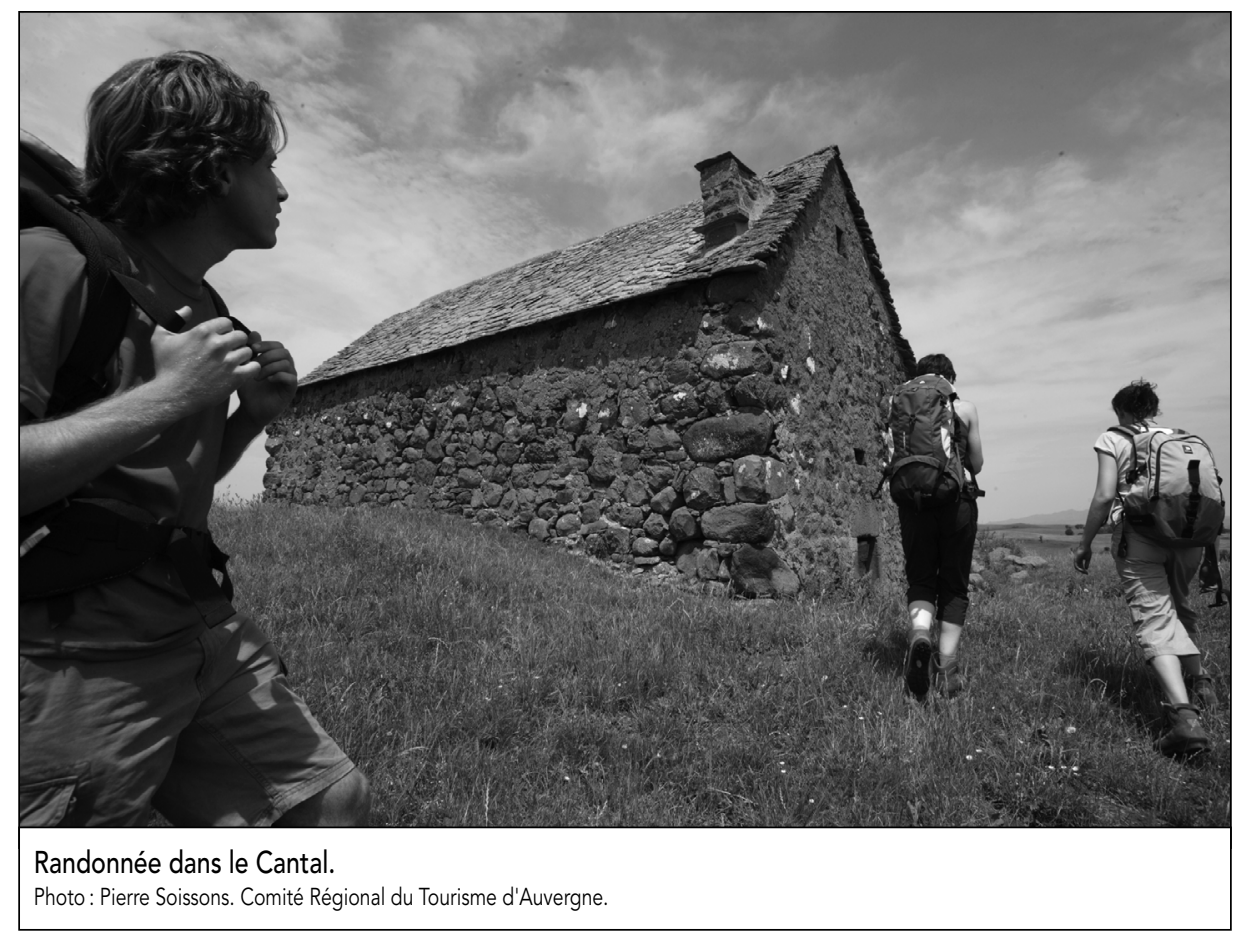

profil n'est pas aussi simple. De vrais entrepreneurs combineront les deux produits, attirant des clientèles complémentaires. Gîtes et chambres pourront apporter l'un ou l'autre un complément de revenu pourvu qu'ils soient de qualité et gérés comme de véritables produits touristiques. Les hébergeurs les plus professionnels (profils " complément de revenu » ou "entreprise ") gèrent aussi bien des chambres d'hôtes que des gîtes.

Le seul constat solide relève que les hébergeurs à logique patrimoniale disposent très généralement de gîtes.

\section{Apports de l'analyse quantitative}

Qu'apporte l'analyse quantitative de la population d'hébergeurs touristiques? L'enquête téléphonique auprès de 300 propriétaires gestionnaires n'a pas permis de retrouver les nuances qu'avait relevé l'entretien semi-directif en tête à tête. Le profil "complément de revenu " se fond dans les profils «patrimoine " et " projet de vie ». De même, le profil «patrimoine-épargne » ne se distingue plus du profil « patrimoine-passion ».

L'analyse quantitative met en relief l'importance du profil patrimoine pris globalement: $59 \%$ du total des acteurs. Ce groupe dominant représente l'archétype du petit propriétaire traditionnel peu professionnel.
Le $41 \%$ restant correspond aux prestataires pour lesquels l'hébergement touristique rural constitue le projet de vie : un groupe d'acteurs beaucoup plus professionnels renouvelant la population des hébergeurs (un sur deux est néo-rural). Par ailleurs, 25\% du total sont des prestataires pour lesquels la qualité de vie est essentielle. Leur projet est partagé entre qualité de vie et activité rémunératrice. Finalement, $16 \%$ des acteurs conduisent, en vrais professionnels, une entreprise touristique à part entière.

La perte de nuances consentie au traitement statistique, imputable à la méthode d'entretien téléphonique, est compensée par une approche qualifiée de la diversité. Décidément, le secteur de l'hébergement touristique révèle une forte spécificité : seulement $16 \%$ de véritables entreprises, un quart des acteurs en quête d'un projet de vie multifonctionnel, une forte prépondérance d'hébergeurs traditionnels gérant leur patrimoine dans un cadre plus domestique que marchand.

Face à l'avenir, les acteurs envisagent principalement continuer leur activité sans grands changements: les deux tiers des hébergeurs pensent poursuivre leur activité durant les cinq à dix prochaines années ; le quart sont dans l'indécision; $8 \%$ envisagent d'arrêter, pour la retraite principalement. 
Les hypothèses que nous avions formulées reliant dynamisme des acteurs, esprit d'entreprise et avenir de la structure, semblent infirmées par les discours. Rien ne permet de dire que les entrepreneurs se projettent plus dans la pérennisation de leur structure que les passionnés du patrimoine ou les tenants de la qualité de vie. Seule confirmation banale : les $18 \%$ d'hébergeurs qui envisagent de développer leur affaire appartiennent principalement au groupe des entrepreneurs touristiques.

\section{Apports de l'analyse localisée dynamique}

L'approche globale qualitative et évolutive du parc d'hébergement touristique rural ainsi que l'approche quantitative par profil de la population d'hébergeurs contribuent à décrire des objets (parc d'hébergement, hébergeurs) et à révéler des volumes, des proportions, des mouvements d'ensemble. Cependant de telles descriptions ou analyses peuvent occulter des réalités et des processus qu'un changement d'échelle aurait révélés. On a pu ainsi constater que la trajectoire d'évolution générale du parc d'hébergement de gîtes du Massif central était contredite dans certains départements. II a paru utile d'explorer plus finement les mouvements d'évolution saisis par les soldes entrées-sorties du parc d'hébergement par une analyse spatiale dynamique localisée.

Loin de constituer une étude exhaustive couvrant l'espace Massif central et la durée 1980-2004, cet éclairage approfondit le processus de renouvellement des structures d'hébergement, en relevant les flux d'entrées et de sorties du parc, unité par unité, sur deux départements, Creuse et Puy de Dôme, sur la période 2000-2004.

Alors que l'analyse des soldes entrées-sorties de 2000 à 2004 donne pour la Creuse une perte nette de 14 gîtes sur un total de 364 en 2000 et pour le Puy de Dôme une perte nette de 1 gîte sur 460 la même année, l'analyse des flux montre sur la période 84 créations et 98 disparitions en Creuse, 117 créations et 118 disparitions dans le Puy de Dôme. En quatre ans, le quart des structures du parc se trouvent donc renouvelées!

Qui plus est, la cartographie communale sur la période 2000-2004 fait état d'une redistribution spatiale des structures d'hébergement à l'occasion de ce renouvellement.
L'analyse localisée apporte ainsi un éclairage nouveau - qui mériterait sans doute d'être confirmé - sur le changement réel que les données globales traduisaient par une lente évolution. Le renouvellement des acteurs, et des profils qu'il induit vraisemblablement, est sans doute lourd de conséquences pour l'avenir.

\section{L'avenir du parc d'hébergement touristique rural}

L'objet de l'étude prospective est d'imaginer les avenirs possibles du parc d'hébergement touristique rural du Massif central.

La démarche prospective s'est particulièrement développée en France depuis les années 1950 dans le contexte de la planification centralisée, puis ultérieurement sous l'impulsion de la DATAR, et au-delà avec la décentralisation des régions... Le centre de ressources prospectives de la DIACT ${ }^{3}$ comptabilise 160 études prospectives en 2006, nationales ou régionales, sectorielles ou globales, sur des sujets aussi divers que l'emploi, la démographie, l'environnement, les dynamiques territoriales...

La prospective n'est ni la planification, ni la programmation, encore moins la prévision. II s'agit au fond d'une attitude d'esprit, que Pierre Masse, ancien commissaire au Plan, qualifie d'« indiscipline intellectuelle », néanmoins formalisée par un ensemble de méthodes qui permettent, comme le dit Gaston Berger (1967), "de voir loin, large et profond ». Les auteurs dans la lignée d'Henri de Jouvenel (1993) lui confèrent trois missions: comprendre le présent, anticiper les changements, ouvrir le champ des possibles. Les avancées les plus récentes contribuent à outiller la démarche prospective en vue d'une utilisation stratégique et opérationnelle par les apports de la modélisation et du calcul informatique (Godet, 1997).

La connaissance des évolutions passées, des profils d'hébergeurs, des stratégies déployées et des intentions exprimées dans les discours permet d'entrevoir un mouvement d'ensemble dans le sillage du passé, des pratiques et des intentions des acteurs actuels. Le parc continue d'évoluer en nombre d'unités et en qualité; les acteurs du changement, les hébergeurs, se renouvellent; l'univers du marché touristique, des campagnes, des organisations professionnelles, de l'intervention publique changent.
La troisième partie, s'appuyant sur les propos d'experts interrogés sur la question de l'avenir du parc d'hébergement rural du Massif central, dessine des scénarios d'avenir à dix ans. Treize experts ont apporté leur contribution à la réflexion ${ }^{4}$. En raison de leur position (experts du ministère du Tourisme, représentants professionnels, chercheurs et consultants), ils situent leur analyse principalement à l'échelle nationale, mais trois d'entre eux apportent, par leur appartenance territoriale, un éclairage particulier sur le Massif central.

La synthèse dégage successivement les tendances lourdes qui orientent l'évolution, des tendances plus récentes ou encore incertaines. De ces tendances les variables motrices qui formatent l'évolution sont extraites, les facteurs de rupture susceptibles de changer la donne sont isolés. Les différents scénarios issus de ces entretiens identifient des solutions de remplacement. Quelques réflexions stratégiques concluent l'analyse.

\section{Les tendances lourdes}

D'une façon générale, et depuis plus d'une décennie, l'attractivité de la campagne se confirme (Kayser, 1993; Hervieu et Viard, 1996; Perrier-Cornet, 2002). Elle se traduit par un mouvement d'installation résidentiel (résidences principales et secondaires) et une fréquentation touristique croissante de la campagne ${ }^{5}$. Le tourisme rural profite de ce mouvement, entrant de plain-pied dans l'ère du marché.

La demande touristique pour la campagne, en relation avec l'accroissement du temps libre, est en progression continue, dans le contexte de courts séjours notamment. Cette progression est soutenue par l'afflux des clientèles étrangères du nord de l'Europe (Allemagne, Belgique, Pays-Bas, Royaume-Uni).

Cette demande s'adresse à la fois à l'espace de la nature et à celui de la ruralitée ${ }^{\text {. Les tou- }}$ ristes cherchent le calme et la tranquillité de la campagne (en regard au stress urbain), la sécurité physique et alimentaire, source de bonne santé (le bon air, de bons produits de terroir, la sécurité...), l'esthétique (paysage, belle architecture, vieilles pierres...), le ressourcement et la convivialité. Les touristes sont attachés à la symbolique rurale : la ferme, le village, le clocher, les paysages agraires.

D'un autre point de vue, ces touristes attendent de leur lieu d'accueil le confort et le haut de gamme (et, pour les gîtes ou chambres d'hôtes, de plus en plus le trois épis). 


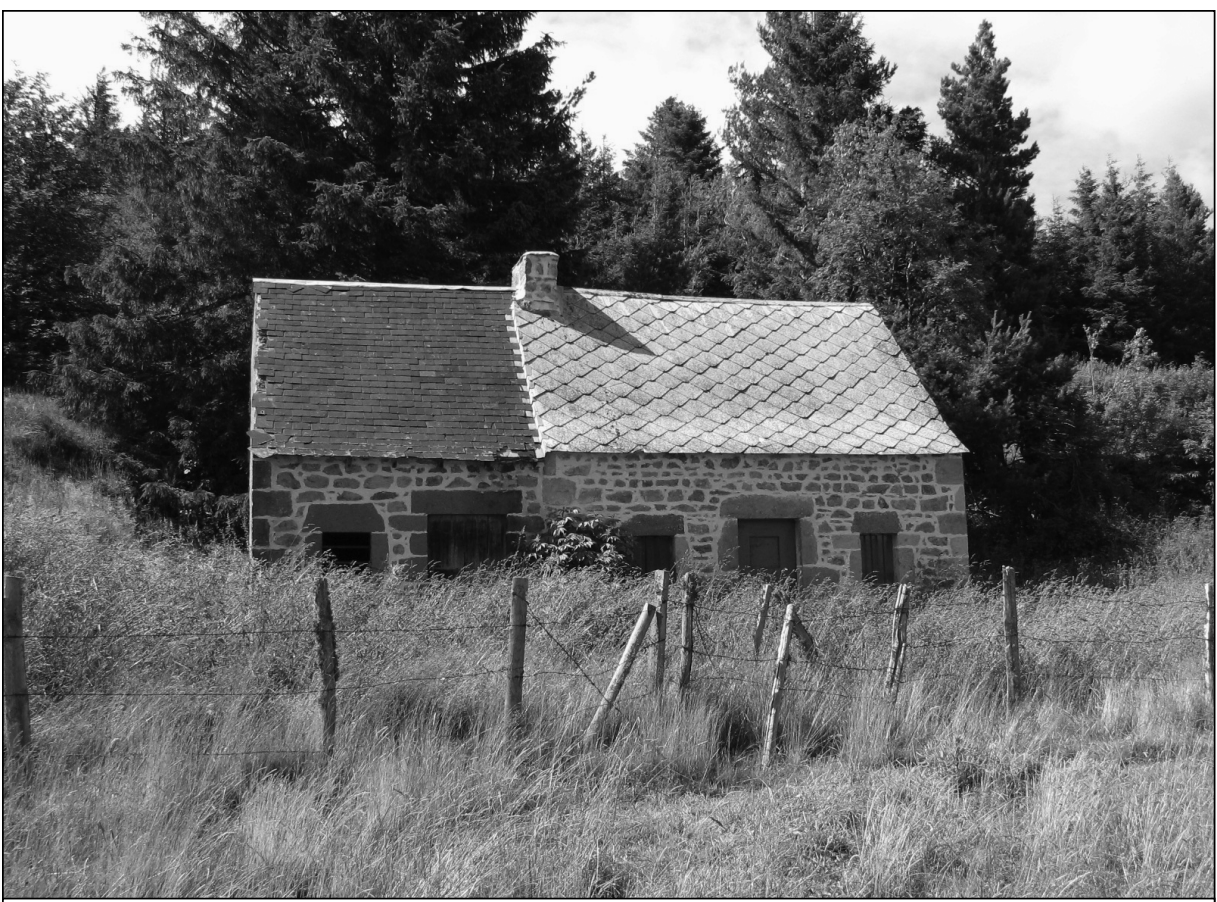

Un buron d'estives à réouvrir aux visiteurs. Puy de Dôme.

Source: collection personnelle, Jean-François Mamdy.

La notion de qualité est primordiale pour les touristes. Cela signifie de plus en plus d'équipements modernes, comme dans la vie quotidienne, de services (garde d'enfants, ménage compris pour la fin du séjour), un accueil personnalisé, mais aussi des facilités de réservation, beaucoup de souplesse (location de week-end ou de quelques jours), la possibilité de réserver à la dernière minute, ainsi que la fiabilité (l'intérêt d'une marque comme Gîte de France).

Le niveau de revenu de la clientèle des hébergements ruraux, qui se situe de moyen à élevé, est progressivement plus élevé 7 . Avec la demande de qualité et l'encouragement des pouvoirs publics qui soutiennent financièrement la politique de haut de gamme, les prix montent, sélectionnant les segments de clientèle à haut pouvoir d'achat et attisant la concurrence avec des destinations alternatives moins chères.

La clientèle touristique de la campagne, parce qu'elle voyage partout, est de plus en plus avertie. Non seulement elle compare les prix et les qualités, elle cherche à discuter les prix, elle devient consumériste et procédurière (dénonciation des contrats).

L'hébergement rural est au cœur de l'offre touristique rurale, avant même la destination et les activités. En relation avec une demande de qualité - architecture, confort, services -, l'offre d'hébergement rural se diversifie. La concurrence s'intensifie avec la multiplication d'initiatives issues de grands groupes (résidences de tourisme), de filières (hébergement de plein air de qualité, relais-châteaux) ou de particuliers (villages de gîtes...).

L'évolution du parc d'hébergement rural est en proie à des forces contraires : d'un côté un appauvrissement de structures par absence de réinvestissement, de l'autre un renouvellement avec des créations, des innovations, des offres toujours plus complètes autour de l'hébergement : accueil, animation, services et activités. La tendance à l'enrichissement de l'offre varie selon les segments de clientèles. La demande de services semble générale, la demande d'activités plus particulière (rando-accueil).

Les gîtes et les chambres d'hôtes relèvent, aux dires des experts, de deux formes assez différentes d'hébergement touristique, de conception et de contenu de séjour touristique. Le gîte offre le côté pratique, fonctionnel, familial, éventuellement collectif. La chambre d'hôte relève d'une pratique plus intimiste, de couple, itinérante. Le gîte s'inscrit dans la tradition, la chambre dans la modernité comme une nouvelle formule d'hô- tellerie à la campagne: pratique, d'autant qu'elle est souvent associée à la table d'hôte, souple, intime et relationnelle. Les tendances actuelles soulignent l'engouement pour cette formule en pleine expansion, qui est en phase avec la demande de qualité (les trois épis dominent à Gîtes de France) et de tourisme « intelligent », curieux de découvrir les provinces françaises et leurs habitants. Les gîtes, quant à eux, restent une formule stable, toujours demandée.

Dans la lignée des distinctions précédentes, les propriétaires se scindent entre anciens et nouveaux. Les propriétaires de gîtes à logique patrimoniale, souvent âgés, sont les plus passifs. Pour une part importante, ils s'inscrivent dans la routine et n'investissent plus dans le gîte, ce qui en affecte la qualité. À l'inverse, les propriétaires de chambres, de plus en plus nombreux, sont souvent entreprenants (projet de vie) ; ils disposent de moyens financiers (étrangers, cadres supérieurs) et recherchent une rentabilité économique.

Cette catégorisation rencontre pour une part l'évolution régressive de la population traditionnelle d'hébergeurs, les agriculteurs, premiers gestionnaires des gîtes ruraux. Cependant, au sein même des agriculteurs, on trouvera des entrepreneurs agritouristiques dynamiques (le nouveau guide conjoint Bienvenue à la ferme / Gîtes de France atteste de ce dynamisme). De même, chez les propriétaires gestionnaires patrimoniaux, dont l'effectif est assez stable, l'abandon des uns est compensé par l'arrivée de nouveaux propriétaires, passionnés de campagne et de vieilles pierres, disposant de moyens financiers, soucieux de rentabiliser leur investissement ou de faire des placements financiers sécuritaires.

Ces analyses tendancielles ont une résonance pour l'ensemble de la campagne française, dont le Massif central est un peu l'archétype. Les trois experts plus particulièrement positionnés sur le Massif central soulignent le potentiel de développement d'un espace déjà bien mis en valeur, mais susceptible d'attirer davantage, autour des figures de la nature préservée, des grands espaces et de la ruralité, de ses fermes d'accueil. Ils s'expriment sur cet espace avec optimisme, du fait de son désenclavement récent, de son attrait nouveau, d'acteurs dynamiques et ouverts à l'innovation (le Massif central est souvent considéré comme un laboratoire d'expérimentation...). 


\section{Les tendances nouvelles}

Les tendances nouvelles constituent des signaux discrets pour la réflexion prospective. Le regard des experts permet d'identifier trois champs d'analyse : la concurrence / complémentarité entre résidents et touristes, la stratégie des nouveaux hébergeurs, le comportement des nouvelles clientèles du tourisme rural.

L'attractivité de la campagne française encourage beaucoup d'étrangers, mais aussi de cadres urbains, à acheter de l'immobilier traditionnel pour résidence principale ou secondaire. Cet investissement revêt aussi un caractère de placement dans la pierre. Le prix du bâti de caractère s'envole, rendant l'investissement en hébergement touristique toujours plus coûteux. Cette concurrence entre résidentiel et touristique joue plutôt contre le développement du locatif touristique, mais en même temps elle peut aussi favoriser l'usage touristique transitoire.

Les nouveaux hébergeurs, souvent nouveaux arrivants de racines citadines, parfois d'origine étrangère, apparaissent beaucoup plus autonomes que les anciens. Ils sont animés à la fois par un projet de vie et un souci de rentabilité économique. Plus professionnels, ils se développent et évoluent à l'écoute de leurs clientèles. Demandeurs de conseils professionnels, ils sollicitent davantage les institutions, les bousculent et s'affranchissent éventuellement des réseaux historiques, quitte à s'organiser en collectif autonome.

Dans le prolongement des tendances lourdes qui soulignaient le changement des clientèles du tourisme rural (recherche de confort, de services, de qualité...), il convient de noter des tendances nouvelles : ainsi, le caractère exigeant et zappeur des clientèles influencées par les modèles de tourisme industriel « tout, tout de suite » du Club Méditerranée, le loisir en toute sécurité dans la bulle protectrice des centre parks, le côté voyeur et curieux chez Disney... Beaucoup expriment le goût de la nature sauvage ou domestiquée, dont l'expression la plus aboutie est le tourisme écologique ou écologiste. Certains attendent des séjours différents dans le contexte d'un tourisme affinitaire, entre amis partageant une passion commune (chorale, motards...), ou d'un tourisme familial adapté aux nouvelles retrouvailles des familles éclatées.
L'offre d'hébergement est donc appelée à se diversifier en intégrant de nouveaux concepts, tels les villages de gîtes avec services et multiples activités, mais aussi en qualifiant ses produits face à la concurrence de grands organisateurs de voyages ou de l'hôtellerie, ou encore d'autres réseaux éventuellement étrangers.

En contrepoint de cette évolution sociétale se pose la question des clientèles modestes, qui constituaient le fond de commerce du tourisme rural aux origines. Ne risquent-elles pas d'être mises à l'écart? D'autant que les porteurs de projets, encouragés par les collectivités territoriales, semblent peu enclins à développer une offre touristique pour clientèles à revenu modeste. Ce segment de marché semblerait pourtant rentable, sous réserve d'une bonne gestion.

\section{Les variables motrices}

Les variables motrices de l'évolution du parc d'hébergement rural relèvent de trois domaines : les attentes sociétales, la stratégie des acteurs, les politiques publiques.

L'attrait des campagnes, de la nature et de la ruralité constitue un puissant vecteur de fréquentation touristique. L'attente des clientèles est subordonnée à un comportement consumériste, exigeant en qualité, en variété, en fiabilité... L'obligation de qualité est générale et concerne l'ensemble des clientèles. La demande se déplace vers les produits haut de gamme. Les clientèles de plus en plus expérimentées réclament des produits et services nouveaux, élaborés et variés. Si la promesse n'est pas tenue, elles deviennent facilement procédurières. Cependant, sous réserve d'une offre multiforme appropriée, cet engouement est susceptible d'alimenter une économie touristique à forte valeur ajoutée.

La dynamique de l'offre touristique d'hébergements ruraux dépend de l'aptitude des hébergeurs à construire l'offre attendue. Le renouvellement des acteurs est de bon augure dans la mesure où les porteurs de projets sont souvent dynamiques, motivés par le patrimoine et la rentabilité, qu'ils disposent de fonds suffisants et se comportent en entrepreneurs. Faut-il encore que les dispositifs d'incitation financière, de formation et d'animation soient en place, accessibles et efficients. Faut-il aussi que les acteurs s'organisent sur les territoires et dans les filières.
A contrario, une partie importante des hébergeurs semble aujourd'hui peu disposée à évoluer, pour des raisons de mentalité, de manque de professionnalisme ou simplement de désintérêt à conduire une activité économique. Le poids relatif des hébergeurs traditionnels est donc facteur d'inertie.

II est enfin probable que la disqualification des hébergeurs traditionnels face à la concurrence de nouvelles destinations et de nouveaux produits et la difficile transmission de l'outil de travail ralentiront les évolutions et le renouvellement du parc.

Les aides publiques jouent à coup sûr un rôle important dans l'évolution de l'offre d'hébergements rural. En la matière, la raréfaction des crédits européens fait reposer sur les départements et les régions le soutien aux investissements. L'inventaire de dispositifs d'aide mis en place par les conseils généraux et régionaux montre une forte variabilité entre collectivités sur les niveaux et les conditions d'attribution. II est certain que les stratégies des collectivités territoriales auront des répercussions importantes sur le développement en effectif et en qualité des structures touristiques.

D'un autre point de vue, les politiques publiques peuvent aussi inciter les hébergeurs à s'organiser, à progresser en qualité, à améliorer leur mise en marché, à dénoncer un certain nombre de problèmes, mais elles peuvent aussi faire peser des contraintes réglementaires, fiscales, sociales, difficilement supportables pour les hébergeurs.

L'Internet continuera de jouer un rôle important dans le développement du parc d'hébergement rural, au même titre que toutes les infrastructures qui rendent les campagnes plus accessibles aux clientèles. Le rôle des pouvoirs publics est là aussi important pour accompagner les acteurs dans leur modernisation.

\section{Les facteurs de rupture}

Des villes plus attractives, plus durables, diminueraient sans doute l'attrait de la campagne pour les résidents permanents, secondaires ou touristiques, ainsi que l'engouement pour l'hébergement rural.

Une probable cause de rupture dans la progression du parc d'hébergement tient à la disponibilité du bâti. L'attrait de la campagne 
favorise les usages résidentiels non marchands (résidences principales et secondaires) au détriment de l'usage touristique marchand.

Dans le même sens, la multiplication des contraintes administratives, réglementaires, fiscales et sociales décourage l'initiative de porteurs de projets d'hébergement rural.

Une autre source de rupture dans les tendances favorables à l'hébergement rural relève de la concurrence. D'une part le haut de gamme tire les prix vers le haut, d'autre part la concurrence fait rage autour de nouveaux concepts d'hébergement à la campagne, tel Huttopia $^{8}$, ou de nouvelles offres touristiques patrimoniales en Europe méditerranéenne et atlantique ou à l'Est ${ }^{9}$.

D'aucuns pensent (Gîtes de France par exemple) que l'authenticité, jointe à la qualité, sera garante de l'attractivité de l'hébergement rural.

Un autre élément-clé pour l'avenir de l'hébergement rural tient aux aides publiques. On sait que les aides européennes vont se tarir. Les départements et les régions assureront-ils le relais? Le rôle des subventions publiques sur la création et la réhabilitation du parc d'hébergement ne sont plus à démontrer. Qu'en sera-t-il si, dans certaines régions ou départements, celles-ci venaient à se tarir? En l'absence de progression en qualité, la concurrence pourrait alors s'intensifier, au détriment du parc.

La dynamique des acteurs reste déterminante pour l'avenir. Si l'entrée de nouveaux porteurs de projet se poursuit, le parc évoluera encore en qualité et en innovation. Que feront les agriculteurs toujours moins nombreux globalement, mais peut-être plus ouverts à la multifonctionnalité et au tourisme et contraints par une politique agricole de moins en moins protectrice à rechercher d'autres sources de revenus?

\section{Scénarios d'avenir}

La méthode des scénarios (Godet, 1997) repose sur différentes analyses - analyse structurelle des variables-clés, des stratégies d'acteurs, analyse morphologique du champ des possibles - permettant de caractériser des situations d'avenir, puis de discriminer, parmi les multiples combinaisons possibles, les configurations les plus cohérentes et probables. Les configurations en question relèvent à la fois du contexte général de la société et du contexte propre au tourisme, général et rural en particulier.

À l'occasion de la réflexion prospective sur le parc d'hébergement touristique rural, nous avons ainsi construit quatre scénarios d'avenir contrastés de tourisme rural, sur la base des tendances identifiées, des variables motrices et des facteurs de rupture.

\section{Le tourisme de luxe à la campagne}

Le tourisme de luxe à la campagne répond à une demande de qualité croissante. Tourisme de niche haut de gamme, il concerne des clientèles d'origines française et étrangère, mais exclut les catégories de clientèles à revenu modeste. Les gîtes bas de gamme disparaissent lentement, retournant à l'usage résidentiel.

Les nouveaux propriétaires gestionnaires d'origine citadine aisée, parfois étrangers, disposent de compétences étendues et de moyens financiers personnels pour créer des hébergements touristiques. Ils sont ou deviennent des professionnels avertis.

Les collectivités publiques (région / département) poursuivent leur politique d'incitation financière, dans l'idée de promotion d'une image de qualité.

L'évolution du parc d'hébergement rural du Massif central épouse partiellement le scénario du tourisme de luxe. On sait que les gîtes trois épis représentaient $23 \%$ du parc en 1990, $39 \%$ en 2000 et $42 \%$ en 2004. Par ailleurs, près des deux tiers des chambres d'hôtes avaient le niveau de qualité trois épis en 2000. Les nouveaux propriétaires hébergeurs qui partagent projet de vie et projet d'entreprise sont dans cette logique de haut de gamme. Les propriétaires patrimoniaux peuvent être séduits par cette montée en gamme pour rentabiliser leur structure en l'améliorant durablement ; les propriétaires gestionnaires en recherche de compléments de revenu, pour partie également, sous réserve d'être incités à le faire et accompagnés par la collectivité. Notons que certains départements ont clairement opté pour cette voie : la Haute-Vienne, la Corrèze, l'Aveyron. Les aides à l'investissement sont aiguillées vers les gîtes et les chambres trois épis.

\section{Le tourisme de campagne en retrait}

Dans un contexte où les villes retrouvent peu à peu une qualité de vie (villes durables) et une attractivité, le parc d'hébergement rural est concurrencé par des filières industrielles de tourisme (résidence de tourisme) qui adoptent des concepts innovants, par des offres d'hébergement rural attractives à l'étranger ou par d'autres types d'hébergement concurrents (hôteliers, para hôteliers...). L'offre d'hébergement spécifique au tourisme rural décline.

Les acteurs patrimoniaux, qui sont les plus nombreux, abandonnent leur effort de réhabilitation, de mise aux normes, de requalification, de modernisation. Seuls les plus innovants parviennent à échapper à la concurrence en jouant sur des ressources spécifiques (produits de terroir, produits authentiques, mise en scène...) et en se positionnant sur des niches commerciales.

L'aide publique spécifique à l'agriculture et au monde rural s'efface dans les pays de l'Ouest européen, aggravant le retrait du tourisme rural.

Dans le cas du Massif central, la tendance à la rétraction du parc de gîtes est déjà avérée dans neuf départements sur 19. Ce sont principalement les gîtes modestes qui disparaissent. Cependant, en même temps, un renouvellement conséquent se produit et les soldes départementaux légèrement négatifs cachent une importante vague de renouvellement.

L'appui des collectivités publiques peut être significatif pour endiguer le recul d'une partie du parc, notamment auprès des propriétaires sensibles au complément de revenu apporté par la location touristique.

\section{Le Tourisme de campagne typique}

Le scénario de la campagne typique met en valeur la spécificité, la diversité, la qualité des produits du tourisme rural. L'idée dominante est «la qualité à tous les étages ». On cherche à développer des produits authentiques et spécifiques. On explore toute la gamme d'hébergement (du un au cinq épis), à travers toutes les formes de tourisme, pour tous les types de clientèles. L'appui des collectivités publiques se concentre sur l'authenticité des hébergements ruraux et des produits touristiques : le cas du département de l'Aude, aux marges méditerranéennes du Massif central, qui décline son identité cathare dans toute la gamme des produits touristiques 
en est une anticipation de plus abouties. Tous les acteurs, quelle que soit leur logique, sont incités de fait à suivre une voie qui rencontre la demande des clientèles touristiques de court séjour. La diversité des profils d'hébergeurs est prise en compte et en main dans une démarche de qualité qui valorise l'ensemble des aménités rurales.

Le Massif central dispose d'un potentiel considérable en termes de diversité de terroirs et de paysages. Les hébergements ruraux et les produits qui leurs sont associés ont les qualités requises pour satisfaire la demande d'authenticité, de typicité, d'esthétique, de confort... sous réserve que les pouvoirs publics soutiennent cet effort.

L'aide des collectivités publiques pourrait logiquement soutenir les initiatives des acteurs qui visent un développement de l'hébergement de caractère et de qualité. Le scénario de " la qualité à tous les étages » suppose en revanche un redéploiement des actions (cahier des charges, animation, formation, conseil, appui financier...) et des cibles vers toutes les gammes de produits et de clientèles.

\section{Le tourisme rural en réseau}

Le tourisme rural s'organise au sein de filières et de réseaux. Les organisations d'acteurs, encouragées par les collectivités publiques, poussent les hébergeurs à adopter des chartes de qualité qui répondent à la demande de confort, de services, d'animation, de développement durable... Le mouvement s'étend à toutes les échelles de territoire (un parc naturel régional, un département, une région, la France, voire l'Europe....) et concerne les principales filières : Gîtes de France / Eurogîtes, Bienvenue à la Ferme, Accueil paysan, Gîtes Panda...

Le tourisme rural se structure en réseaux et en marques, évoluant d'une certaine façon vers la standardisation. Les clientèles, de plus en plus consuméristes, s'adressent aux exploitants organisés commercialement, qui leur fournissent des prestations ad hoc.

Depuis quelques années, des initiatives décisives d'organisation de l'offre touristique rurale et de mise en marché sont à l'œuvre en Auvergne. Le Massif central semble à son tour s'organiser pour porter l'offre diffuse des territoires sur les marchés urbains et étrangers. Ce processus s'appuie effectivement sur les réseaux professionnels et territoriaux et les filières, imposant une certaine standardisation des produits (marques et labels). Le scénario des réseaux est sans doute prometteur pour le tourisme rural et ses hébergements, mais il est difficile et coûteux. II suppose une participation active des acteurs à la base, des contributions financières plus importantes de leur part, un repositionnement des fédérations (Gîtes de France / Clévacances), un partenariat public / privé clarifié. Dans cette phase de construction, les pouvoirs publics ne peuvent se désintéresser de la question.

Si les quatre scénarios décrits constituent des caricatures d'avenir contrastées, ils traduisent néanmoins des processus en cours. lls trouveront ici ou là une part d'application et de réalité. Cependant, les dynamiques actuelles donnent probablement davantage de poids aux deux derniers scénarios décrits. L'organisation des acteurs est en effet devenue le mot d'ordre principal dans le monde du tourisme rural français. Les réseaux d'acteurs s'imposent dans tous les domaines, à commencer par la promotion et la commercialisation. À titre d'exemple, la région Auvergne est en pointe sur le plan national pour organiser la promotion des territoires touristiques, leur mise en marché, mais aussi la production touristique à l'échelle d'agences locales de tourisme. Par ailleurs, dans un contexte de concurrence de plus en plus vive entre destinations, la carte de la typicité apparaît de jour en jour comme l'avantage comparatif du tourisme à la campagne.

\section{Conclusion}

L'avenir n'est pas écrit ; il existe en fait des marges de manœuvre. En effet, malgré l'apparence d'une évolution plutôt lente, caractérisée par une demande soutenue évoluant vers une gamme élevée de qualité et une offre prédéterminée par le profil des propriétaires (dominante d'hébergeurs âgés), le parc connaît des mouvements internes considérables d'entrées et de départs. Le fort renouvellement des hébergements permet de penser que les changements sont très ouverts et que les institutions peuvent largement peser sur l'évolution en influençant le choix des propriétaires gestionnaires, particulièrement ceux des profils patrimoniaux, les plus nombreux.

L'encouragement des collectivités publiques est en effet décisif, non pas tant pour la décision de faire de l'hébergement rural ou non que pour influencer certaines orientations vers la qualité, la mise en marché plus performante, le recours à des modes d'organisation en réseau, les propriétaires gestionnaires étant en effet de plus en plus avisés et opportunistes.

Dans un monde incertain, les gîtes et les chambres d'hôtes sont sans doute, quel que soit le profil d'hébergeur, une forme de placement sans risque, susceptible d'apporter un revenu. La recherche de la performance n'est pas l'idéal de la majorité, mais la recherche d'un «plus » est appréciée par tous. Si les aides apportées par les collectivités et leurs réseaux permettent ce "plus", nul doute qu'il contribuera à faire progresser le parc d'hébergement.

Parmi les différents scénarios, la combinaison des deux derniers semble la plus souhaitable pour le pays, l'aménagement du territoire et les clientèles. Ces scénarios dessinent la nature des encouragements que les institutions pourraient mettre en œuvre: la progression de la qualité autour de valeurs de spécificité et de durabilité, sur toute la gamme et pour toutes les clientèles, la mise en réseau pour une meilleure organisation de l'offre et de la mise en marché.

Gîtes de France jouit d'une situation de quasi-monopole; c'est le premier exploitant européen pour les hébergements ruraux. Cependant, cette position est aujourd'hui disputée par une concurrence interne (Clévacances...) et externe (propositions européennes).

D'une part, Gîtes de France devra s'affirmer dans sa capacité à satisfaire une demande spécifique aux clientèles du tourisme rural, d'authenticité, d'activité, de qualité et de sécurité face aux réseaux concurrents. II lui faudra apporter la preuve de sa supériorité en matière de dynamique de réseau, de qualité du produit, de mise en marché.

D'autre part, Gîtes de France devra remobiliser et «refidéliser » ses adhérents, notamment les nouveaux propriétaires si différents des anciens membres, d'origine agricole ou rurale. Peut être faudra-t-il inventer une nouvelle culture de réseau?

L'étude évolutive et prospective a permis une clarification des données quantitatives et qualitatives sur le parc d'hébergement rural de Gîtes de France du Massif central (gîtes et chambres) et son évolution passée ou future. 
Elle a mis en évidence les difficultés à recueillir une information atomisée, hétérogène et partiellement inaccessible. L'information collectée sur le parc d'hébergement reste partielle, même si Gîtes de France reste le principal réseau. Elle souligne des tendances globales, mais montre aussi que ces tendances sont différentes selon les régions ou les départements.

Par ailleurs, elle indique dans sa dimension prospective que les possibles sont multiples, voire contradictoires, et que les évolutions peuvent toujours être infléchies, notamment par des politiques publiques volontaristes.

Parmi les pistes de travail intéressantes, notons l'intérêt à mieux cerner les flux entrées / sorties d'hébergeurs qui renseignent autrement sur le changement que les soldes par territoire. Notons aussi l'intérêt d'avoir une vision et une analyse spatiale des évolutions, du parc, des hébergeurs, des politiques des collectivités publiques.

Jean-François Mamdy est professeur au Département territoire et société, Unité mixte de recherches Métafort, ÉNITA de Clermont-Ferrand, France.

Marion Guillot est ingénieure d'études à I'ÉNITA de Clermont-Ferrand, France.

Nathalie Disez est maître de conférences au Département territoire et société, Unité mixte de recherches Métafort, ÉNITA de Clermont-Ferrand, France.

\section{Notes}

1 DATAR: Délégation à l'aménagement du territoire et à l'action régionale.

SPOT Auvergne : Système permanent d'observation du tourisme en Auvergne.

NFAER: Nouvelles fonctions d'accueil de l'espace rural, équipe intégrée à l'Unité mixte de recherches Métafort.

Métafort : Mutation des activités, des espaces et des formes d'organisation dans les territoires ruraux.

ÉNITA : École nationale d'ingénieurs des travaux agricoles.

2 À l'échelle nationale en 2002, on compte 42 300 gîtes et 26000 chambres d'hôtes. Clévacances rassemble 24000 meublés et 3400 chambres en 2005, dans les divers espaces touristiques, campagne comprise.
En 2004, Accueil Paysan regroupe 450 structures d'accueil et Bienvenue à la Ferme 330 fermes de séjour.

3 Nouvel acronyme de la DATAR: Délégation interministérielle à l'aménagement et à la compétitivité des territoires.

4 Les experts interrogés sont: S. Brault, J.C. Bontron, P. Correze-Lénée, J.F. Crolat, V. Daniel, J. de Caffarelli, M. Guérin, J. Laurens, N. le Scouarnec, C. Lespinasse-Tarabat, M. Quéméré, L. Pommaret et C. Tijou.

5 Les comptes du tourisme (Direction du tourisme / Suivi des déplacements touristiques des Français / 1999) indiquent une progression de $18 \%$ des nuitées marchandes des résidents français à la campagne de 1990 à 1998. Sur la même période (Direction du tourisme/ Enquête aux frontières / 1999), la progression des nuitées touristiques des étrangers (non-résidents) à la campagne est estimée à $96 \%$ !

Le Mémento du Tourisme 2005 (Ministère du Tourisme de France) donne une augmentation de $16 \%$ de l'ensemble des séjours et des nuitées des Français à la campagne de 2000 à 2004.

6 ODIT (Observation, développement et ingénierie touristiques) France (2005 : 36-63) décrit, dans son Carnet de route de la campagne et de la moyenne montagne, les qualités et les comportements des clientèles du tourisme rural de nos jours.

7 L'ouvrage d'ODIT France (2005 : 38-39) souligne la prééminence des clientèles de bon niveau de revenu, confirmée par l'élévation continue de la gamme de qualité des offres que notre étude sur les hébergements touristiques a fait ressortir.

8 Huttopia est un concept d'hébergement inspiré de Thomas More (1516) : "un lieu qui n'est dans aucun lieu, un ailleurs nostalgique... ". II s'agit en fait d'une marque commerciale d'hébergements rustiques, dans des lieux "sauvages ", mais en France! visant à rompre avec le quotidien, à déconnecter du monde, à revenir aux sources...

9 Les programmes européens Leader ont permis en une douzaine d'années un développement fulgurant d'hébergements touristiques ruraux patrimoniaux dans les pays voisins de la France (Espagne, Portugal, Grèce, Irlande...). L'élargissement de l'Union européenne encourage désormais à l'Est les initiatives de développement touristique à partir du patrimoine rural.

\section{Bibliographie}

"Le Tourisme Rural: Acteurs, Clients, Produits", Source, hors série, n 5 , juillet 2005 .

Berger, Gaston (1967), Étapes de la Prospective, Paris, Presses universitaires de France.

Boltansky, Laurent, et Luc Thévenot (1991), De la justification; Les économies de la grandeur, Paris, Gallimard.
Boltansky, Laurent, et Luc Thévenot (1998), « Les valeurs en question; À l'épreuve des grands principes ", Sciences humaines, no 79, p. 19-25.

Charron, Isabelle (2003), Diversifier par l'agrotourisme : étude exploratoire sur la prise de décision du producteur agricole, Faculté d'agriculture, Université Laval, Québec.

de Jouvenel, Henri (1993), «Sur la méthode prospective: un bref guide méthodologique", Futuribles, n 179 , septembre.

Disez, Nathalie (1996), Agritourisme et développement territorial: Exemples dans le Massif central, thèse de doctorat de géographie, Université Blaise Pascal, Clermont-Ferrand.

Disez, Nathalie (1999), «Agritourisme: Iogiques d'acteurs et logiques de territoire ? ", Économie Rurale, n 250, mars-avril, p. 40-46.

Gallo, Solenne (2004), État des lieux et perspectives de l'hébergement touristique rural en Massif central, mémoire de diplôme d'études supérieures spécialisées (DESS) en développement rural, Université Lumière, Lyon.

Godet, Michel (1997), Manuel de prospective stratégique. Tome 1: Une indiscipline intellectuelle, Paris, Dunod.

Godet, Michel (1997), Manuel de prospective stratégique. Tome 2: L'art et la méthode, Paris, Dunod.

Hervieu, Bertrand, et Jean Viard (1996), Au bonheur des campagnes, Paris, L'Aube.

Kayser, Bernard (1993), Naissance de nouvelles campagnes, Paris, DATAR / L'Aube.

Loudiyi, Salma (2003), Les hébergements touristiques dans les campagnes d'Auvergne: une contribution à l'analyse et à l'étude dynamique du tourisme en espace rural, thèse de doctorat de géographie, Université Blaise Pascal, Clermont-Ferrand.

Mamdy, Jean-François, Nathalie Diez, et Monique Begon (2001), «Agritourisme et territoire: Le cas du Massif central », Téoros, vol. 20, n 2 , p. 44-51.

ODIT France (2005), Carnet de route de la campagne et de la moyenne montagne, la demande, l'offre et les recommandations marketing, Paris, ODIT France.

Perret, Jacques, et Emmanuelle Marcepoil (2001), L'agritourisme en 2001, diversité des territoires et des acteurs: entre témoignage et commerce, Rapport du Cemagref / Ministère de l'agriculture.

Perrier-Cornet, Philippe (dir.) (2002), Repenser les campagnes, Paris, DATAR / L'Aube.

Simon, Anthony (2000), La pluriactivité dans l'agriculture des montagnes françaises ; Étude géographique, thèse de doctorat de géographie, Université Blaise Pascal, Clermont-Ferrand.

Simmoneaux, Jean (1999), Acteurs, enjeux et régulations dans la dynamique du tourisme rural, thèse de doctorat de géographie, Université de Toulouse-Le Mirail. 